\title{
PERIODIC SOLUTIONS FOR A DIFFERENTIAL EQUATION IN BANACH SPACE \\ BY
}

JAMES H. LIGHTBOURNE III

Abstract. Suppose $X$ is a Banach space, $\Omega \subset X$ is closed and convex, and $A:[0, \infty) \times \Omega \rightarrow X$ is continuous. Then if

$$
\lim _{h \rightarrow 0+}|x+h A(t, x) ; \Omega| / h=0 \text { for all }(t, x) \in[0, \infty) \times \Omega,
$$

there exist approximate solutions to the initial value problem

(IVP)

$$
u^{\prime}(t)=A(t, u(t)), \quad u(0)=x \in \Omega .
$$

In the case that $A(t, x)=B(t, x)+C(t, x)$, where $B$ satisfies a dissipative condition and $C$ is compact, we obtain a growth estimate on the measure of noncompactness of trajectories for a class of approximate solutions. This estimate is employed to obtain existence of periodic solutions to (IVP).

Let $X$ be a Banach space with norm $|\cdot|$ and $D \subset X$ be closed and convex. Suppose $B:[0, \infty) \times D \rightarrow X$ is continuous and for each $x \in D$ there exists a unique solution $u(\cdot ; x):[0, \infty) \rightarrow D$ to the initial value problem:

$$
u^{\prime}(t)=B(t, u(t)), \quad u(0)=x .
$$

Define the translation operator $U:[0, \infty) \times D \rightarrow D$ associated with $(0.1)$ by $U(t, x)=u(t ; x)$. Let $X^{*}$ with norm also denoted $|\cdot|$ be the dual space of $X$ and for $x, y \in X$ define $\langle x, y\rangle_{-}=\inf \left\{\phi(x): \phi \in X^{*}, \phi(y)=|y|^{2}=|\phi|^{2}\right\}$. Then if for some real number $L$

$$
\langle B(t, x)-B(t, y), x-y\rangle_{-}\left\langle L|x-y|^{2}\right.
$$

for all $(t, x),(t, y) \in[0, \infty) \times D$, the translation operator associated with (0.1) satisfies $|U(t, x)-U(t, y)| \leqslant e^{L t}|x-y|$. Thus if $L<0$ and $\sigma>0$ there exists unique $x_{\sigma} \in D$ for which $U\left(\sigma, x_{\sigma}\right)=x_{\sigma}$. Consequently, if $B$ is $\sigma$-periodic in $t$, then there exists a unique $\sigma$-periodic solution $u$ to $(0.1)$ with $u(0)=x_{\sigma}$. In fact, such results are available when, for each $t, B(t, \cdot)$ is possibly multivalued (Crandall and Pazy [2]).

Let $C$ be completely continuous and suppose for each $x \in D$ there is a unique solution to the perturbed problem:

Received by the editors September 2, 1976.

AMS (MOS) subject classifications (1970). Primary 34G05; Secondary 47H15.

Key words and phrases. Banach space, dissipative, measure of noncompactness, periodic solutions. 
(IVP)

$$
u^{\prime}(t)=B(t, u(t))+C(t, u(t)), \quad u(0)=x \in D .
$$

In general, the translation operator associated with (IVP) is not Lipschitz continuous. However, under certain conditions the translation operator associated with (IVP) does satisfy estimates involving the measure of noncompactness. The measure of noncompactness of a bounded subset $F$ of $X$, denoted $\alpha[F]$, is the infimum of $\varepsilon>0$ such that $F$ can be covered by a finite number of sets of diameter no larger than $\varepsilon$. In $\S 1$ rather than assume the existence of solutions to (IVP) and consider trajectories of solutions, we obtain a measure of noncompactness estimate on the trajectories of approximate solutions to (IVP). We also consider a condition on $B$ more general than (0.2). In $\$ 2$ these estimates are applied to the existence of periodic solutions and in $\$ 3$ applications and an example are indicated.

1. Measure of noncompactness estimates. In this section we obtain growth estimates on the measure of noncompactness along trajectories of approximate solutions to the initial value problem:

$$
u^{\prime}(t)=B(t, u(t))+C(t, u(t)), \quad u(0)=z \in D .
$$

The concept of the measure of noncompactness is attributed to Kuratowski [6]. More recently, the measure of noncompactness has been applied to existence of solutions to differential equations in Banach spaces (see, for example, Cellina [1]; Li [9]; and Martin [11], [12]). We list without proof several properties of the measure of noncompactness.

Proposition 1. Suppose $E_{1}$ and $E_{2}$ are bounded subsets of $X$.

(i) If $E_{1} \subset E_{2}$, then $\alpha\left[E_{1}\right] \leq \alpha\left[E_{2}\right]$;

(ii) $\alpha\left[E_{1}\right]=\alpha\left[\bar{E}_{1}\right]$, where $\bar{E}_{1}$ is the closure of $E_{1}$;

(iii) $\alpha\left[E_{1}\right]=0$ if and only if $E_{1}$ is totally bounded;

(iv) $\alpha\left[E_{1}+E_{2}\right] \leqslant \alpha\left[E_{1}\right]+\alpha\left[E_{2}\right]$, where $E_{1}+E_{2}=\left\{x+y: x \in E_{1}, y \in\right.$ $\left.E_{2}\right\}$;

(v) $\alpha\left[\operatorname{co}\left(E_{1}\right)\right]=\alpha\left[E_{1}\right]$, where $\operatorname{co}\left(E_{1}\right)$ denotes the convex hull of $E_{1}$.

Throughout this paper let $\psi:[0, \infty) \times R \rightarrow \mathbf{R}$ be a continuous function such that scalar equation

$$
\tau^{\prime}(t)=\psi(t, \tau(t)), \quad \tau(0)=r \in[0, \infty)
$$

has the property that for $r=0$ there is only the trivial solution and for $r>0$ the maximal solution $\tau(t ; r)$ exists on $[0, \infty)$. Also, we shall frequently assume the following:

(E1) $D \subset X$ is closed, bounded, and convex and $B$ and $C$ are continuous functions from $[0, \infty) \times D$ into $X$;

(E2) $\lim _{h \rightarrow 0+}|x+h[B(t, x)+C(t, x)] ; D| / h=0$ for all $(t, x) \in[0, \infty)$ $\times D$, where, for $y \in X,|y ; D|=\inf \{|y-z|: z \in D\}$; 
(E3) $B$ is bounded on bounded subsets of $[0, \infty) \times D$ and $\langle B(t, x)-$ $B(t, y), x-y\rangle_{-} \leqslant \psi(t,|x-y|)|x-y|$ for all $(t, x),(t, y) \in[0, \infty) \times D$;

(E4) $C$ maps bounded subsets of $[0, \infty) \times D$ into totally bounded subsets of $X$;

(E5) Either (i) $C$ is uniformly continuous on $[0, \infty) \times D$ or (ii) the dual space $X^{*}$ of $X$ is uniformly convex.

In the following theorem we define approximate solutions to (IVP) and give sufficient conditions for their existence. For the proof of Proposition 2 see Martin [10].

Throughout this paper we let $\left\{\varepsilon_{n}\right\}_{1}^{\infty}$ be a nonincreasing sequence in $(0,1)$ with $\lim _{n \rightarrow \infty} \varepsilon_{n}=0$.

Proposition 2. Suppose (E1) and (E2) hold. Let $z \in D$. Then for each $T>0$ there exists $M=M(T, z)>0$ such that for each positive integer $n$ there is a continuous function $u_{n} ;[0, T] \rightarrow D$ with the properties:

(1) $u_{n}(0)=z$;

(2) $\left|u_{n}(t)-u_{n}(s)\right| \leqslant M|t-s|$ for all $t, s \in[0, T]$; and

(3) for all but a finite number of $t \in[0, T], u_{n}^{\prime}(t)$ exists and $\mid u_{n}^{\prime}(t)-$ $\left[B\left(t, u_{n}(t)\right)-C\left(t, u_{n}(t)\right)\right] \mid \leqslant \varepsilon_{n}$.

Furthermore, if $\left\{u_{n}\right\}_{1}^{\infty}$ satisfies (1)-(3) and $\lim _{n \rightarrow \infty} u_{n}(t)=u(t)$ for each $t \in[0, T]$, then $u$ is continuous from $[0, T]$ into $D$ and satisfies (IVP) for all $t \in[0, T]$.

Remark 1. If $B+C$ is bounded on bounded subsets of $[0, \infty) \times D, M$ in Proposition 2 may be chosen independently of $z$; i.e., for each $T>0$ there exists $M=M(T)>1$ such that the family of functions

$$
\mathcal{Q}(z, n, T, M)=\{u:[0, T] \rightarrow D: u \text { satisfies (1)-(3) of Proposition 2 }\}
$$

is nonempty for all $z \in D$ and each positive integer $n$.

Recall that $\tau(t ; r)$ denotes the maximal solution to (IVP) $)_{\mathrm{s}}$ on $[0, \infty)$ with $\tau(0 ; r)=r$.

THEOREM 1. Suppose (E1) - (E5) hold and $T>0$. Choose $M=M(T)>0$ and for each $z \in D$ and positive integer $n$ define $\mathcal{Q}(z, n, T, M)$ as in Remark 1. Then for each $E \subset D$ there exists a sequence of continuous functions $\tau_{n}(\cdot ;$ $\alpha[E]):[0, T] \rightarrow \mathbf{R}$ such that

$$
\lim _{n \rightarrow \infty} \tau_{n}(t ; \alpha[E])=\tau(t ; \alpha[E])
$$

uniformly on $[0, T]$ and for sufficiently large $n$

$$
\alpha[\{u(t): u \in \mathbb{Q}(z, n, T, M) \text { with } z \in E\}] \leqslant \tau_{n}(t ; \alpha[E])
$$

for all $t \in[0, T]$.

Two lemmas are essential to our proof of Theorem 1 . The proof of the first 
lemma can be found in Lakshmikantham and Leela [7].

LemMa 1. Let $r \geqslant 0$. For each $T>0$ there exists $\beta(T)>0$ such that if $0<\beta<\beta(T)$ then the maximal solution $\tau_{\beta}(t ; r)$ to

$$
\tau^{\prime}(t)=\psi(t, \tau(t))+\beta, \quad \tau(0)=r+\beta,
$$

exists on $[0, T]$. Furthermore, $\lim _{\beta \rightarrow 0+} \tau_{\beta}(t ; r)=\tau(t ; r)$ uniformly on $[0, T]$, where $\tau(t ; r)$ is the maximal solution to (IVP).

LEMMA 2. Let $r \geqslant 0$ and choose $\beta>0$ such that the maximal solution $\tau_{\beta}(t$; $r)$ to $(1.1)$ exists on $[0, T]$. Then the maximal solution $\theta_{\beta}(t ; r)$ to

$$
\theta^{\prime}(t)=2 \psi\left(t, \theta(t)^{1 / 2}\right) \theta(t)^{1 / 2}+2 \beta \theta(t)^{1 / 2}, \quad \theta(0)=(r+\beta)^{2},
$$

exists on $[0, T]$ and $\theta_{\beta}(t ; r)=\left[\tau_{\beta}(t ; r)\right]^{2}$.

Proof of Lemma 2. The assertion readily follows from the observation that if $\theta$ is a solution to (1.2) then $\theta(t) \neq 0$ for all $t \in[0, T]$.

Let $\mathcal{C}([a, b] ; X)$ denote the Banach space of continuous functions $u$ from $[a, b]$ into $X$ with norm $\|\cdot\|$ defined by $\|u\|=\sup \{|u(t)|: a \leqslant t \leqslant b\}$. Also, we use the facts that if $x, y, z \in X$ then $\langle x+y, z\rangle_{-}\left\langle\langle x, z\rangle_{-}+|y||z|\right.$ and if $u:[a, b] \rightarrow X$ is differentiable at $t \in(a, b)$ with $p(t)=|u(t)|^{2}$ then $p_{-}^{\prime}(t)$ exists with $p_{-}^{\prime}(t)=2\left\langle u^{\prime}(t), u(t)\right\rangle_{-}$.

Proof of Theorem 1. For $E \subset D$ and positive integer $n$ let $Q(E ; n)=$ $\cup_{z \in E} \mathbb{Q}(z, n, T, M)$. Suppose (E5(i)) holds; i.e., $C$ is unformly continuous on $[0, \infty) \times D$. Let $m$ be a positive integer. Since $\mathcal{Q}(E ; m)$ is an equicontinuous family of $\mathcal{C}([0, T] ; X)$ and $C$ is uniformly continuous, the family $\{C(\cdot, u(\cdot))$; $u \in \mathbb{Q}(E ; m)\}$ is equicontinuous. Also, by (E4) $\{C(t, u(t)): u \in \mathbb{Q}(E ; m)$ and $t \in[0, T]\}$ is totally bounded on $X$. By Ascoli's Theorem there exist a positive integer $l$ and a finite subset $\left\{u_{1}, \ldots, u_{l}\right\}$ of $\mathbb{Q}(E ; m)$ such that for each $u \in \mathbb{Q}(E ; m)$ there is an $i \in\{1, \ldots, l\}$ for which $\| C(\cdot, u(\cdot))$ $C\left(\cdot, u_{i}(\cdot)\right) \| \leqslant \varepsilon_{m} / 2$. For each $1 \leqslant i \leqslant l$ let

$$
Q_{i}=\left\{u \in \mathbb{Q}(E ; m):\left\|C(\cdot, u(\cdot))-C\left(\cdot, u_{i}(\cdot)\right)\right\| \leqslant \varepsilon_{m} / 2\right\} .
$$

Let $\left\{E_{i}\right\}_{1}^{k}$ be a finite cover of $E$ for which the diameter of each $E_{i}$ is less that $\alpha[E]+\varepsilon_{m}$. Let $R_{i j}=\mathscr{Q}\left(E_{i}, m\right) \cap Q_{j}$ and note that for each $t \in[0, T]$ the sets $\left\{u(t): u \in R_{i j}\right\}, 1 \leqslant i \leqslant k$ and $1 \leqslant j \leqslant l$, form a finite cover for $\{u(t)$ : $u \in \mathbb{Q}(E ; m)\}$.

Let $1 \leqslant i \leqslant k$ and $1 \leqslant j \leqslant l$ and $u, v \in R_{i j}$. Define $p:[0, T] \rightarrow[0, \infty)$ by $p(t)=|u(t)-v(t)|^{2}$. Then $p$ is continuous and for all but a finite number of $t \in[0, T] p_{-}^{\prime}(t)$ exists with 


$$
\begin{aligned}
p_{-}^{\prime}(t)= & 2\left\langle u^{\prime}(t)-v^{\prime}(t), u(t)-v(t)\right\rangle_{-} \\
\leqslant & 2\langle B(t, u(t))+C(t, u(t))-B(t, v(t))-C(t, v(t)), u(t)-v(t)\rangle_{-} \\
& +\varepsilon_{m}|u(t)-v(t)| \\
\leqslant & 2\langle B(t, u(t))-B(t, v(t)), u(t)-v(t)\rangle_{-}+2 \varepsilon_{m}|u(t)-v(t)| \\
\leqslant & 2 \psi(t,|u(t)-v(t)|)|u(t)-v(t)|+2 \varepsilon_{m}|u(t)-v(t)| .
\end{aligned}
$$

Also, $p(0) \leqslant\left(\alpha[E]+\varepsilon_{m}\right)^{2}$ and thus by Lemma $1, p(t) \leqslant \theta_{e_{m}}(t ; \alpha[E])$ for all $t \in[0, T]$, where $\theta_{\varepsilon_{m}}(t ; \alpha[E])$ is the maximal solution to (1.2) with $\beta=\varepsilon_{m}$ and $r=\alpha[E]$. Consequently, by Lemma $2, p(t)^{1 / 2} \leqslant \tau_{\varepsilon_{m}}(t ; \alpha[E])$ and thus

$$
\sup \left\{|u(t)-v(t)|: u, v \in R_{i j}\right\} \leqslant \tau_{\varepsilon_{m}}(t ; \alpha[E])
$$

for each $t \in[0, T]$. In addition, $\cup_{i, j} R_{i j}=\mathbb{Q}(E ; m)$ and therefore by the definition of the measure of noncompactness

$$
\alpha[\{u(t): u \in \mathbb{Q}(E ; m)\}] \leqslant \tau_{\varepsilon_{m}}(t ; \alpha[E])
$$

for all $t \in[0, T]$. By Lemma $1, \lim _{m \rightarrow \infty} \tau_{\varepsilon_{m}}(t, \alpha[E])=\tau(t ; \alpha[E])$ uniformly on $[0, T]$, and the assertion follows if (E5(i)) holds.

Suppose (E5(ii)) holds (i.e., the dual space $X^{*}$ of $X$ is uniformly convex) and let $m$ be a positive integer. For each $u \in \mathbb{Q}(E ; m)$ define $W_{u}:[0, T] \rightarrow X$ by $W_{u}(t)=\int_{0}^{t} C(s, u(s)) d s$. By (E4) we have the family $\left\{W_{u}: u \in \mathbb{Q}(E ; m)\right\}$ is equicontinuous and the set $\left\{W_{u}(t): u \in \mathbb{Q}(E ; m)\right.$ and $\left.t \in[0, T]\right\}$ is totally bounded. Let $\delta>0$. By Ascoli's Theorem there exists a finite set $\left\{u_{i}\right\}_{1}^{l} \subset$ $\mathbb{Q}(E ; m)$ such that for each $u \in \mathbb{Q}(E ; m)$ there exists $1 \leqslant i \leqslant l$ with $\| W_{u}-$ $W_{u_{i}} \| \leqslant \delta / 2$. For $1 \leqslant i \leqslant l$ let

$$
Q_{i}=\left\{u \in \mathbb{Q}(E ; m):\left\|W_{u}-W_{u_{i}}\right\| \leqslant \delta / 2\right\} .
$$

Let $\left\{E_{i}\right\}_{1}^{k}$ be a finite cover of $E$ such that the diameter of each $E_{i}$ is less than $\alpha[E]+\varepsilon_{m}$. As before, let $R_{i j}=\mathbb{Q}\left(E_{i} ; m\right) \cap Q_{j}$ and let $u, v \in R_{i j}$ for some $1 \leqslant i \leqslant k$ and $1 \leqslant j \leqslant l$. Define $p:[0, T] \rightarrow[0, \infty)$ by

$$
p(t)=\left|u(t)-v(t)-W_{u}(t)+W_{v}(t)\right|^{2} .
$$

For all but a finite number of $t \in[0, T], p_{-}^{\prime}(t)$ exists with

$$
\begin{aligned}
& p_{-}^{\prime}(t)=2\left\langle u^{\prime}(t)-v^{\prime}(t)-C(t, u(t))+C(t, v(t))\right. \\
& \left.u(t)-v(t)-W_{u}(t)+W_{v}(t)\right\rangle_{-} \\
& <2\left\langle B(t, u(t))-B(t, v(t)), u(t)-v(t)-W_{u}(t)+W_{v}(t)\right\rangle_{-} \\
& \quad+4 \varepsilon_{m} p(t)^{1 / 2}
\end{aligned}
$$

Let $\varepsilon>0$. By the uniform continuity of the map $(x, y) \rightarrow\langle x, y\rangle_{-}$on bounded subsets of $X \times X$ (see Kato [3]) and by the uniform continuity of $\psi$ on compact subsets of $[0, \infty] \times[0, \infty)$ we may choose $\delta>0$ such that 


$$
\begin{aligned}
p_{-}^{\prime}(t) & \leqslant 2\langle B(t, u(t))-B(t, v(t)), u(t)-v(t)\rangle_{-}+\varepsilon / 2+4 \varepsilon_{m} p(t)^{1 / 2} \\
& \leqslant 2 \psi(t,|u(t)-v(t)|)|u(t)-v(t)|+\varepsilon / 2+4 \varepsilon_{m} p(t)^{1 / 2} \\
& \leqslant 2 \psi\left(t, p(t)^{1 / 2}\right) p(t)^{1 / 2}+4 \varepsilon_{m} p(t)^{1 / 2}+\varepsilon .
\end{aligned}
$$

By Lemmas 1 and 2 the maximal solution $\theta_{4 \varepsilon_{m}, \varepsilon}(t ; \alpha[E])$ to the equation

$$
\theta^{\prime}(t)=2 \psi\left(t, \theta(t)^{1 / 2}\right) \theta(t)^{1 / 2}+4 \varepsilon_{m} \theta(t)^{1 / 2}+\varepsilon, \quad \theta(0)=\alpha[E]+\varepsilon_{m},
$$

exists on $[0, T]$ for sufficiently large $m$ and small $\varepsilon$. Therefore, we have

$$
p(t)=\left|u(t)-v(t)-W_{u}(t)+W_{v}(t)\right|^{2} \leqslant \theta_{4 \varepsilon_{m}, \varepsilon}(t ; \alpha[E])
$$

for all $t \in[0, T]$. By Lemma $1, \lim _{\varepsilon \rightarrow 0+} \theta_{4 \varepsilon_{m}, \varepsilon}(t ; \alpha[E])=\theta_{4 \varepsilon_{m}}(t ; \alpha[E])$ uniformly on $[0, T]$, where $\theta_{4 \varepsilon_{m}}(\cdot ; \alpha[E])$ is the maximal solution to (1.2) with $\beta=4 \varepsilon_{m}$. Consequently, for any $\varepsilon>0$ and $1 \leqslant i \leqslant k$ and $1 \leqslant j \leqslant l$

$$
\sup \left\{\left|u(t)-W_{u}(t)-v(t)+W_{v}(t)\right|: u, v \in R_{i j}\right\} \leqslant\left[\theta_{4 \varepsilon_{m \varepsilon} \varepsilon}(t ; \alpha[E])\right]^{1 / 2}
$$

for each $t \in[0, T]$ and thus

$$
\alpha\left[\left\{u(t)-W_{u}(t): u \in \mathbb{Q}(E ; m)\right\}\right] \leqslant\left[\theta_{4 \varepsilon_{m}}(t ; \alpha[E])\right]^{1 / 2}
$$

for each $t \in[0, T]$. Noting that $\left\{W_{u}(t): u \in \mathbb{Q}(E ; m)\right.$ and $\left.t \in[0, T]\right\}$ is totally bounded we have for each $t \in[0, T]$ that

$$
\begin{aligned}
\alpha[\{u(t): u \in \mathbb{Q}(E ; m)\}] & =\alpha\left[\left\{u(t)-W_{u}(t): u \in \mathbb{Q}(E ; m)\right\}\right] \\
& \leqslant\left[\theta_{4 \varepsilon_{m}}(t ; \alpha[E])\right]^{1 / 2}=\tau_{4 \varepsilon_{m}}(t ; \alpha[E]) .
\end{aligned}
$$

By Lemma $1, \lim _{m \rightarrow \infty} \tau_{4 \varepsilon_{m}}(t ; \alpha[E])=\tau(t ; \alpha[E])$ uniformly on $[0, T]$, and the assertions follow.

We say that $u$ is a solution to (IVP) on $[0, b)$ provided $u:[0, b) \rightarrow D$ and $u$ is continuous on $[0, b)$ and satisfies (IVP) for each $t \in[0, b)$.

Theorem 2. Let (E1) - (E5) hold. Then for each $z \in D$ there exists a solution to (IVP). Furthermore, noncontinuable solutions to (IVP) exist on $[0, \infty)$ and for each $E \subset D$

$$
\alpha[\{u(t): u \text { is a solution to (IVP), } u(0) \in E\}] \leqslant \tau(t ; \alpha[E])
$$

for all $t \in[0, \infty)$. In addition, suppose for each $z \in D$ there is a unique solution $u(t ; z)$ to (IVP). Then solutions depend continuously on initial values; i.e., if $\left\{z_{n}\right\}_{1}^{\infty} \subset D$ with $\lim _{n \rightarrow \infty} z_{n}=z$, then $\lim _{n \rightarrow \infty} u\left(t ; z_{n}\right)=u(t ; z)$ uniformly on compact subsets of $[0, \infty)$.

Proof. Let $z \in D$ and $T>0$. By Proposition 2 there exists $M>1$ such that $\mathcal{Q}(z, n, T, M)$ is nonvoid for each $n$. By Theorem 1 and since $\alpha[\{z\}]=$ 0 , there exists a sequence of functions $\tau_{n}:[0, T] \rightarrow[0, \infty)$ such that $\lim _{n \rightarrow \infty} \tau_{n}(t)=\tau(t ; 0)=0$ uniformly on $[0, T]$ and 


$$
\alpha[\{u(t): u \in \mathbb{Q}(z, n, T, M)\}] \leqslant \tau_{n}(t)
$$

for each $t \in[0, T]$. Select a sequence $\left\{u_{n}\right\}_{1}^{\infty}$ with $u_{n} \in \mathbb{Q}(z, n, T, M)$ for each $n$. Then $\lim _{n \rightarrow \infty} \alpha\left[\left\{u_{k}(t): k \geqslant n\right\}\right]=0$ uniformly on $[0, T]$ and thus for each $t \in[0, T]$ the set $\left\{u_{n}(t)\right\}_{1}^{\infty}$ is totally bounded. Also, by (2) of Proposition 2 $\left\{u_{n}\right\}_{1}^{\infty}$ is an equicontinuous family on $[0, T]$ and therefore by Ascoli's Theorem there is a uniformly convergent subsequence of $\left\{u_{n}\right\}_{1}^{\infty}$, which by Proposition 2 converges to a solution of (IVP).

Suppose $u$ is a noncontinuable solution to (IVP) defined on $[0, c)$ with $c<\infty$. (Note that by the preceding paragraph we have local existence of solutions and thus the noncontinuable solution $u$ is defined on $[0, c)$ rather than $[0, c]$.) Then

$$
|u(t)-u(s)|=\left|\int_{s}^{t} B(r, u(r))+C(r, u(r)) d r\right| \leqslant|t-s| M_{1}
$$

where $M_{1}=\sup \{|B(t, x)+C(t, x)|:(t, x) \in[0, c] \times D\}$. Therefore, $\lim _{t \rightarrow c^{-}} u(t)=w$ exists and defining $u(c)=w$ we extend $u$ to $[0, c]$, a contradiction to the noncontinuability of $u$.

If $u$ is a solution to (IVP) then $u$ is an $\varepsilon_{n}$-approximate solution for all $n$. Thus by Theorem 1 for $E \subset D$

$$
\alpha[\{u(t): u \text { is a solution to (IVP), } u(0) \in E\}]<\tau(t ; \alpha[E])
$$

for all $t \in[0, \infty)$.

Finally, suppose solutions to (IVP) are unique and let $\left\{z_{n}\right\}_{1}^{\infty} \subset D$ with $\lim _{n \rightarrow \infty} z_{n}=z$. Since $\alpha\left[\left\{z_{n}\right\}_{1}^{\infty}\right]=0$ from Theorem 1 we have $\alpha\left[\left\{u\left(t ; z_{n}\right)\right.\right.$ : $n>1\}]=0$ for each $t \in[0, \infty)$. Let $c>0$. Then the family $\left\{u\left(\cdot ; z_{n}\right): n>1\right\}$ is equicontinuous on $[0, c]$ and so by Ascoli's Theorem $\left\{u\left(\cdot ; z_{n}\right): n>1\right\}$ has a subsequence $\left\{u\left(\cdot ; z_{n_{k}}\right): k \geqslant 1\right\}$ uniformly convergent on $[0, c]$. Let $v(t)=$ $\lim _{k \rightarrow \infty} u\left(t ; z_{n_{k}}\right)$. Then $v$ is continuous on $[0, c]$ and since $\{(r, v(r)): r \in[0, c]\}$ is compact we have

$$
\begin{aligned}
v(t) & =\lim _{k \rightarrow \infty} z_{n_{k}}+\int_{0}^{t} B\left(r, u\left(r ; z_{n_{k}}\right)\right)+C\left(r, u\left(r ; z_{n_{k}}\right)\right) d r \\
& =z+\int_{0}^{t} B(r, v(r))+C(r, v(r)) d r
\end{aligned}
$$

for each $t \in[0, c]$. Since solutions to (IVP) are unique $v(t)=u(t ; z)$ for all $t \in[0, c]$, and continuous dependence follows.

2. Applications to periodic solutions. Let $U$ be the map from $[0, \infty) \times D$ into the class of subsets of $D$ defined by

$$
U(t, x)=\{u(t): u \text { is a solution to (IVP), } u(0)=x\} .
$$

Let $\sigma>0$ and suppose 
(E6) for each $x \in D, B(t, x)+C(t, x)$ is $\sigma$-periodic in $t$.

Then the existence of a $\sigma$-periodic solution to (IVP) is equivalent to the existence of $x \in D$ for which $x \in U(\sigma, x)$. The results of this section rest on this well-known fact and the following fixed point theorem due to Sadovskii [14].

A function $f: E \subset X \rightarrow X$ is said to be condensing if $f$ is continuous on $E$ and for each bounded subset $E_{0}$ of $E$ with $\alpha\left[E_{0}\right]>0$ we have that $\alpha\left[f\left(E_{0}\right)\right]<$ $\alpha\left[E_{0}\right]$. Note that a completely continuous function is condensing.

Proposition 3 (SAdovsKil [14]). Suppose $E \subset X$ is nonempty, closed, bounded, and convex and $f: E \rightarrow E$ is condensing. Then $\{z \in E: f(z)=z\}$ is nonempty and compact.

Let $\mathscr{B} C([0, \infty) ; X)$ be the Banach space of bounded continuous functions $u:[0, \infty) \rightarrow X$ with $\|u\|=\sup \{|u(t)|: t \in[0, \infty)\}$.

THEOREM 3. In addition to (E1)-(E6) suppose solutions to (IVP) are unique. Suppose further that for each $r>0$ the maximal solution $\tau(t ; r)$ to (IVP)s satisfies $\tau(\sigma ; r)<r$. Then the family of $\sigma$-periodic solutions to (IVP) is nonempty and compact in $\Re \mathcal{C}([0, \infty) ; X)$.

Proof. Define $U:[0, \infty) \times D \rightarrow D$ by $U(t, x)=u(t ; x)$ where $u(t ; x)$ is the unique solution to (IVP) with $u(0)=x$. By Theorem $2, U(\sigma, \cdot): D \rightarrow D$ is continuous and by Theorem 2 and the assumption on the maximal solution to $(\mathrm{IVP})_{\mathrm{s}}, U(\sigma, \cdot)$ is condensing. Thus by Proposition 3 the set $\{z \in D: U(\sigma, z)$ $=z\}$ is nonempty and compact from which the assertions follow.

In general, the author does not know if Theorem 3 is valid if solutions to (IVP) are not assumed to be unique. However, we are able to obtain results when the interior of $D$ is nonvoid. The approach involves approximating the right-hand side of (IVP) and for this we consider the scalar equation:

$$
\chi^{\prime}(t)=\psi(t, \chi(t))+\beta \chi(t), \quad \chi(0)=0,
$$

where $\beta>0$.

THEOREM 4. In addition to (E1)-(E6) suppose $D$ has nonempty interior. Suppose further that for each $r>0$ the maximal solution $\tau(t ; r)$ to (IVP)s satisfies $\tau(\sigma ; r)<r$ and for each $\beta>0$ the trivial solution is the maximal solution to (2.1). Then the family of o-periodic solutions to (IVP) is nonempty and compact in $\mathscr{B} \mathcal{C}([0, \infty) ; X)$.

REMARK 2. If $\psi(t, r) \leqslant 0$ for all $(t, r) \in[0, \infty) \times[0, \infty)$ or if $\psi(t, r)=\rho(t) r$ where $\rho:[0, \infty) \rightarrow \mathbf{R}$ is continuous, then the trivial solution is the maximal solution to (2.1).

In the proof of Theorem 4 we use two lemmas. The first lemma may be 
proved by a construction similar to that used by Lasota-Yorke [8]. We omit the proof.

Lemma 3. Let (E1) and (E4) hold and define $R(C)=\{C(t, x):(t, x) \in$ $[0, \sigma] \times D\}$. Then for each $\varepsilon>0$ there exists a continuous function $C_{\varepsilon}:[0, \sigma] \times$ $D \rightarrow X$ satisfying

(i) $\left|C_{\varepsilon}(t, x)-C(t, x)\right| \leqslant \varepsilon$ for all $(t, x) \in[0, \sigma] \times D$;

(ii) $C_{\varepsilon}(t, x) \in$ convex hull of $R(C)$ for all $(t, x) \in[0, \sigma] \times D$;

(iii) $C_{\varepsilon}$ is locally Lipschitz continuous.

Furthermore, if $C$ is uniformly continuous on $[0, \sigma] \times D$ then (iii) may be replaced by

(iii)' $C_{\varepsilon}$ is Lipschitz continuous.

LEMMA 4. In addition to the suppositions of Theorem 4, suppose $\left\{A_{n}\right\}_{1}^{\infty}$ is a sequence of continuous functions defined on $[0, \sigma] \times D$ such that $\lim _{n \rightarrow \infty} A_{n}(t, x)$ $=B(t, x)+C(t, x)$ uniformly on $[0, \sigma] \times D$ and for each $n$ there exists $a$ continuous function $u_{n}:[0, \sigma] \rightarrow D$ with $u_{n}(0)=u_{n}(\sigma)$ and $u_{n}^{\prime}(t)=A_{n}\left(t, u_{n}(t)\right)$ for all $t \in[0, \sigma]$. Thus $\left\{u_{n}\right\}_{1}^{\infty}$ has a subsequence uniformly convergent on $[0, \sigma]$.

Proof of Lemma 4. Let $M>1$ such that

$$
\sup \{|B(t, x)+C(t, x)|:(t, x) \in[0, \sigma] \times D\} \leqslant M-1 .
$$

Since $\lim _{n \rightarrow \infty} A_{n}(t, x)=B(t, x)+C(t, x)$ uniformly on $[0, \sigma] \times D$, we may assume $u_{n} \in \mathbb{Q}\left(u_{n}(0), n, \sigma, M\right)$ for each $n$. For each $n$ define $U_{n}$ from $[0, \sigma] \times$ $D$ into the class of subsets of $D$ by $U_{n}(t, x)=\{u(t): u \in \mathcal{Q}(x, n, \sigma, M)\}$ and $D_{n}=\left\{x \in D: x \in U_{n}(\sigma, x)\right\}$. Then $u_{n}(0) \in D_{n}$ for each $n$. Suppose for the moment that $\lim _{n \rightarrow \infty} \alpha\left[D_{n}\right]=0$. Then $\alpha\left[\left\{u_{n}(0)\right\}_{1}^{\infty}\right]=0$ and thus, by Theorem 1 ,

$$
\lim _{k \rightarrow \infty} \alpha\left[\left\{U_{k}(t, x): x \in\left\{u_{n}(0)\right\}_{1}^{\infty}\right\}\right]=0 \text { for each } t \in[0, \sigma] .
$$

Consequently, for each $t \in[0, \sigma]$ we have $\alpha\left[\left\{u_{n}(t)\right\}_{1}^{\infty}\right]=0$ and therefore the assertion follows from Ascoli's Theorem.

It remains to be shown that $\lim _{n \rightarrow \infty} \alpha\left[D_{n}\right]=0$. Since $D_{n+1} \subset D_{n}, \alpha\left[D_{n}\right]$ is a decreasing sequence of positive numbers and so $\lim _{n \rightarrow \infty} \alpha\left[D_{n}\right]=c$. Suppose for contradiction that $c>0$. By assumption the maximal solution $\tau(t ; c)$ to (IVP) $)_{s}$ satisfies $\tau(\sigma ; c)<c$ and thus $\tau\left(\sigma ; \alpha\left[D_{n}\right]\right)<c$ for sufficiently large $n$. Also, from Theorem 1 and Lemma 1 there exists a sequence of continuous $\tau_{n}\left(\cdot ; \alpha\left[D_{n}\right]\right):[0, \sigma] \rightarrow[0, \infty)$ such that $\lim _{n \rightarrow \infty} \tau_{n}\left(t ; \alpha\left[D_{n}\right]\right)=\tau(t ; c)$ uniformly on $[0, \sigma]$ and we have

$$
\alpha\left[D_{n}\right]=\alpha\left[\left\{U_{n}(\sigma, x): x \in D_{n}\right\}\right] \leqslant \tau_{n}\left(\sigma ; \alpha\left[D_{n}\right]\right)<c \leqslant \alpha\left[D_{n}\right]
$$

for sufficiently large $n$. Since this is impossible we conclude that $\lim _{n \rightarrow \infty} \alpha\left[D_{n}\right]$ $=0$. 
Proof of Theorem 4. Let $\delta_{n} \rightarrow 0+$ and for each $n$ approximate $C$ : $[0, \sigma] \times D \rightarrow X$ with $C_{n}$ satisfying properties (i), (ii), and (iii) or (iii)' of Lemma 3 with $\varepsilon=\delta_{n}$. Let $y_{0}$ be an interior point of $D$ and choose $r>0$ such that $\left\{x \in X:\left|x-y_{0}\right| \leqslant r\right\} \subset D$. For each $n$ define $\lambda_{n}=3 \delta_{n} / r$ and $A_{n}$ : $[0, \sigma] \times D$ by $A_{n}(t, x)=B_{n}(t, x)+C_{n}(t, x)$ where $B_{n}(t, x)=B(t, x)+$ $\lambda_{n}\left(y_{0}-x\right)$. Let $n$ be a positive integer and $(t, x) \in[0, \sigma] \times D$. For each $h>0$ sufficiently small there exists (by (E2)) $x_{h}=x_{h}(h, t, x, n) \in D$ such that $\left|x-x_{h}\right| \leqslant r / 3$ and $\left|x+h[B(t, x)+C(t, x)]-x_{h}\right| \leqslant h \lambda_{n} r / 3$. Thus

$$
\begin{aligned}
\mid x & +h A_{n}(t, x)-\left(1-h \lambda_{n}\right) x_{h}-h \lambda_{n} y_{0} \mid \\
& \quad<\left|x+h(B(t, x)+C(t, x))-x_{h}\right|+h\left|C_{n}(t, x)-C(t, x)\right|+h \lambda_{n}\left|x-x_{h}\right| \\
& <h \lambda_{n} r .
\end{aligned}
$$

It follows that if $z=x+h A_{n}(t, x)$ then $\left|\left(h \lambda_{n}\right)^{-1}\left(z-x_{h}\right)+x_{h}-y_{0}\right|<r$ and thus $\left(h \lambda_{n}\right)^{-1}\left(z-x_{h}\right)+x_{h} \in D$. By the convexity of $D$

$$
z=\left(1-h \lambda_{n}\right) x_{h}+h \lambda_{n}\left[\left(h \lambda_{n}\right)^{-1}\left(z-x_{h}\right)+x_{h}\right] \in D
$$

and, consequently, $\lim _{h \rightarrow 0+}\left|x+h A_{n}(t, x) ; D\right| / h=0$. Also, since for $x, y \in$ $X$ and $a \in \mathbf{R},\langle x+a y, y\rangle_{-}=\langle x, y\rangle_{-}+a|y|$, we have

$$
\begin{aligned}
\left\langle B_{n}(t, x)-B_{n}(t, y), x-y\right\rangle_{-} & =\langle B(t, x)-B(t, x), x-y\rangle_{-}-\lambda_{n}|x-y| \\
& <\psi(t,|x-y|)|x-y| .
\end{aligned}
$$

By Theorem 2 there exists a solution $u:[0, \infty) \rightarrow D$ to

$$
u^{\prime}(t)=B_{n}(t, u(t))+C_{n}(t, u(t)), \quad u(0)=z,
$$

for each $z \in D$. Suppose $z \in D$ and $u, v$ are noncontinuable solutions to (2.2) satisfying $u(0)=z=v(0)$. Let $p(t)=|u(t)-v(t)|^{2}$. Since $C_{n}$ is locally Lipschitz there exist $\beta>0$ and $\varepsilon>0$ such that for each $t \in[0, \varepsilon]$

$$
\begin{aligned}
& p_{-}^{\prime}(t)= 2\left\langle B_{n}(t, u(t))+C_{n}(t, u(t))-B_{n}(t, v(t))\right. \\
&\left.-C_{n}(t, v(t)), u(t)-v(t)\right\rangle_{-} \\
&<2 \psi(t,|u(t)-v(t)|)|u(t)-v(t)|+\beta|u(t)-v(t)|^{2} .
\end{aligned}
$$

Let $q(t)=p(t)^{1 / 2}$. Since the trivial solution is the maximal solution to (2.1) we have by Lemma 2 that $q(t) \equiv 0$ and solutions to (2.2) are unique. By Theorem 3 the suppositions of Lemma 4 hold and by that lemma there exists a solution $u$ to (IVP) with $u(0)=u(\sigma)$. The assertions readily follow.

Theorems 3 and 4 may be applied to existence of critical values when $B+C$ is autonomous.

THEOREM 5. Suppose (E1)-(E5) hold and either solutions to (IVP) are unique or the interior of $D$ is nonvoid. Suppose further that $B(t, x)+C(t, x)=B(x)$ $+C(x)$ for all $(t, x) \in[0, \infty) \times D$ and $\psi(t, r)=\psi(r)$ for all $(t, r) \in[0, \infty)$ 
$\times \mathbf{R}$ with maximal solutions to (IVP) satisfying $\tau(t ; r)<r$ for all $r>0$ and $t \in[0, \infty)$. Let $\theta$ denote the zero of $X$. Then $Z=\{z \in D: B(z)+C(z)=\theta\}$ is nonempty and compact.

Proof. By Theorems 3 and 4 for each positive integer $n$ there exist $x_{n} \in D$ and $u_{n}:[0, \infty) \rightarrow D$ such that $u_{n}$ is a solution to (IVP) with $u_{n}(0)=x_{n}$ and $u_{n}(0)=u_{n}\left(n^{-1}\right)$. Furthermore, since $B+C$ is autonomous we may assume that $u_{n}(0)=u_{n}\left(l n^{-1}\right)$ for all $l \in\{0,1,2, \ldots\}$ and in particular $u_{n}(0)=$ $u_{n}(1)$. Suppose, for contradiction, that $\alpha\left[\left\{x_{n}\right\}_{1}^{\infty}\right]>0$. Then by Theorem 1

$$
\alpha\left[\left\{x_{n}\right\}_{1}^{\infty}\right]=\alpha\left[\left\{u_{n}(1)\right\}_{1}^{\infty}\right] \leqslant \tau\left(1 ; \alpha\left[\left\{x_{n}\right\}_{1}^{\infty}\right]\right)<\alpha\left[\left\{x_{n}\right\}_{1}^{\infty}\right]
$$

which is impossible. Thus $\alpha\left[\left\{x_{n}\right\}_{1}^{\infty}\right]=0$ and there exist $z \in D$ and subsequence $\left\{x_{n_{k}}\right\}_{k=1}^{\infty}$ of $\left\{x_{n}\right\}_{1}^{\infty}$ such that $\lim _{k \rightarrow \infty} x_{n_{k}}=z$. Since for each $k$

$$
u_{n_{k}}(0)=u_{n_{k}}\left(n_{k}^{-1}\right)=u_{n_{k}}(0)+\int_{0}^{n_{k}^{-1}} B\left(u_{n_{k}}(r)\right)+C\left(u_{n_{k}}(r)\right) d r
$$

it follows that $\int_{0}^{n_{k}^{-1}} B\left(u_{n_{k}}(r)\right)+C\left(u_{n_{k}}(r)\right) d r=\theta$ and thus

$$
\begin{aligned}
|B(z)+C(z)| & =\lim _{k \rightarrow \infty}\left|n_{k}^{-1} \int_{0}^{n_{k}^{-1}} B(z)+C(z)-B\left(u_{n_{k}}(r)\right)-C\left(u_{n_{k}}(r)\right) d r\right| \\
& =0 .
\end{aligned}
$$

Therefore $Z$ is nonempty. Suppose, for contradiction, that $\alpha[Z]>0$. Then by Theorem 1

$$
\alpha[Z] \leqslant \tau(1 ; \alpha[Z])<\alpha[Z]
$$

which is impossible. Also, by the continuity of $B+C$ we have $Z$ is closed and thus compact.

3. Applications. In this section we apply our results first to the situation when $X$ is finite dimensional and then to a system of integrodifferential equations.

Suppose $X$ is finite dimensional and $A:[0, \infty) \times D \rightarrow X$ is continuous with $\lim _{h \rightarrow 0+}|x+h A(t, x) ; \quad D| / h=0$ for each $(t, x) \in[0, \infty) \times D$. Since approximate solutions constructed in Proposition 2 are equicontinuous and uniformly bounded, the existence of solutions to the initial value problem

$$
u^{\prime}(t)=A(t, u(t)), \quad u(0)=z \in D,
$$

follows from Ascoli's Theorem. Thus if solutions to (3.1) are unique, the translation operator $U(t, x)$, is defined and continuous on $[0, \infty) \times D$ and $U(\sigma, \cdot)$ leaves $D$ invariant. By the Brouwer Fixed Point Theorem $U(\sigma, \cdot)$ has a fixed point and thus there is a $\sigma$-periodic solution to (3.1) when $A$ is $\sigma$-periodic in $t$. When solutions to (3.1) are not unique but $D$ has nonempty interior, then we employ the approximation techniques of Theorem 4 to obtain existence of periodic solutions. 
Krasnoselskii [5] establishes these finite dimensional results for the special case when $D$ is defined by $D=\left\{x \in \mathbf{R}^{n}: \pi_{i}(x) \leqslant 0\right.$ for all $\left.1 \leqslant i \leqslant k\right\}$ where $\left\{\pi_{i}\right\}_{1}^{k}$ is a (finite) family of continuously differentiable functions from $\mathbf{R}^{n}$ into R.

Suppose $\Lambda$ is a closed cone in $\mathbf{R}^{n}$ (i.e., $\Lambda$ is closed and for each $\xi, \eta \in \Lambda$ and $t, s \geqslant 0$ we have $t \xi+s \eta \in \Lambda$ ). We apply our results to the system of integrodifferential equations:

$$
\begin{aligned}
\frac{\partial u_{i}}{\partial t}(t, s)= & f_{i}\left(t, s, u_{1}(t, s), \ldots, u_{n}(t, s)\right) \\
& +\int_{0}^{1} g_{i}\left(t, s, r, u_{1}(t, r), \ldots, u_{n}(t, r)\right) d r
\end{aligned}
$$

subject to the condition that $u_{i}(0, s)=z_{i}(s)$ and $u(t, s)=$ $\left(u_{1}(t, s), \ldots, u_{n}(t, s)\right) \in \Lambda$.

Let $X=\mathcal{C}\left([0,1] ; \mathbf{R}^{n}\right)$ be the Banach space of continuous functions $x$ from $[0,1]$ into $\mathbf{R}^{n}$ with $|x|=\max \{|x(t)|: 0 \leqslant t \leqslant 1\}$. Let

$$
K(\Lambda)=\{x \in X: x(t) \in \Lambda \text { for all } t \in[0,1]\} .
$$

Conditions on the functions $f=\left(f_{1}, \ldots, f_{n}\right)$ and $g=\left(g_{1}, \ldots, g_{n}\right)$ for application of our results to the system (3.2) are given in the following two lemmas. Let $\langle,\rangle_{-}$be defined as before on the Banach space $\mathbf{R}^{n}$. Note that if $\mathbf{R}^{n}$ has the Euclidean norm then $\langle,\rangle_{-}=\langle$,$\rangle , the usual inner product on \mathbf{R}^{n}$.

LeMMA 5. Let $f:[0, \infty) \times[0,1] \times \Lambda \rightarrow \mathbf{R}^{n}$ be continuous and satisfy

(1) $\left\langle f\left(t, s, \xi_{1}\right)-f\left(t, s, \xi_{2}\right), \xi_{1}-\xi_{2}\right\rangle_{-} \leqslant \psi\left(t,\left|\xi_{1}-\xi_{2}\right|\right)\left|\xi_{1}-\xi_{2}\right|$ for all $(t, s)$ $\in[0, \infty) \times[0,1]$ and $\xi_{1}, \xi_{2} \in \Lambda$;

(2) $\lim _{h \rightarrow 0+}|\xi+h f(t, s, \xi) ; \Lambda| / h=0$ for all $(t, s, \xi) \in[0, \infty) \times[0,1] \times \Lambda$. Define $B$ on $[0, \infty) \times K(\Lambda)$ by $[B(t, x)](s)=f(t, s, x(s))$ for all $s \in[0,1]$. Then $B$ is a continuous function from $[0, \infty) \times K(\Lambda)$ into $X$ which is bounded on bounded subsets of $[0, \infty) \times K(\Lambda)$ and satisfies

(1)' $\left\langle B\left(t, x_{1}\right)-B\left(t, x_{2}\right), x_{1}-x_{2}\right\rangle_{-} \leqslant \psi\left(t,\left|x_{1}-x_{2}\right|\right)\left|x_{1}-x_{2}\right|$ for all $\left(t, x_{1}\right),\left(t, x_{2}\right) \in[0, \infty) \times K(\Lambda) ; \lim _{h \rightarrow 0+}|x+h B(t, x) ; K(\Lambda)| / h=0$ for all $(t, x) \in[0, \infty) \times K(\Lambda)$.

Proof. The proof of the continuity and boundedness of $B$ on $[0, \infty) \times$ $K(\Lambda)$ is straightforward. Let $x_{1}, x_{2} \in \mathcal{C}\left([0,1] ; \mathbf{R}^{n}\right)$ and $t_{0} \in[0,1]$ for which $\left|x_{1}\left(t_{0}\right)-x_{2}\left(t_{0}\right)\right|=\left|x_{1}-x_{2}\right|$. Define $\phi: C\left([0,1] ; \mathbf{R}^{n}\right) \rightarrow \mathbf{R}$ by $\phi(y)=$ $\left\langle y\left(t_{0}\right), x_{1}\left(t_{0}\right)-x_{2}\left(t_{0}\right)\right\rangle_{-}$. Then

$$
\begin{aligned}
\left\langle B\left(t, x_{1}\right)-B\left(t, x_{2}\right), x_{1}-x_{2}\right\rangle_{-} \leqslant \phi\left(B\left(t, x_{1}\right)-B\left(t, x_{2}\right)\right) \\
\quad=\left\langle f\left(t, x_{1}\left(t_{0}\right)\right)-f\left(t, x_{2}\left(t_{0}\right)\right), x_{1}\left(t_{0}\right)-x_{2}\left(t_{0}\right)\right\rangle_{-} \\
\quad \leqslant \psi\left(t,\left|x_{1}\left(t_{0}\right)-x_{2}\left(t_{0}\right)\right|\right)\left|x_{1}\left(t_{0}\right)-x_{2}\left(t_{0}\right)\right|=\psi\left(t,\left|x_{1}-x_{2}\right|\right)\left|x_{1}-x_{2}\right| .
\end{aligned}
$$

See Martin [12] for the proof of (2)'. 
LEMMA 6. Let g: $[0, \infty) \times[0,1]^{2} \times \Lambda \rightarrow \Lambda$ be continuous and define $C$ on $[0, \infty) \times K(\Lambda)$ by

$$
[C(t, x)](s)=\int_{0}^{1} g(t, s, r, x(r)) d r
$$

for all $s \in[0,1]$. Then $C$ is uniformly continuous on bounded subsets of $[0, \infty) \times K(\Lambda)$ and completely continuous from $[0, \infty) \times K(\Lambda)$ into $X$. Furthermore,

$$
\lim _{h \rightarrow 0+}|x+h C(t, x) ; K(\Lambda)| / h=0
$$

for all $(t, x) \in[0, \infty) \times K(\Lambda)$.

Proof. The uniform continuity and complete continuity of $C$ is well-known (see e.g., Krasnoselskii [4]). The remaining assertion follows from the observation that if $x \in K(\Lambda)$ then $C(t, x) \in K(\Lambda)$ and since $K(\Lambda)$ is a cone in $X$ it follows that $x+h C(t, x) \in K(\Lambda)$ for all $h>0$.

For $x, y \in X$ let $\langle x, y\rangle_{+}=\sup \left\{\phi(x): \phi \in X^{*}\right.$ with $\left.\phi(y)=|y|^{2}=|\phi|^{2}\right\}$.

Proposition 4. In addition to the suppositions of Lemmas 5 and 6 suppose there exist $R>0$ such that for $x \in K(\Lambda)$ with $|x|=R$ we have $\langle B(t, x)+$ $C(t, x), x\rangle_{+} \leqslant 0$ for all $t \in[0, \infty)$. Let $K(\Lambda)_{R}=\{x \in K(\Lambda):|x|<R\}$. Then for each $z=\left(z_{i}\right)_{1}^{n} \in K(\Lambda)_{R}$ there exists a solution $u=\left(u_{i}\right)_{1}^{n}:[0, \infty) \rightarrow$ $K(\Lambda)_{R}$ to the system (3.2).

In addition, suppose for each $r>0$ the maximal solution $\tau(t ; r)$ to (IVP)s satisfies $\tau(\sigma ; r)<r$. If $B+C$ is $\sigma$-periodic in $t$ and either solution to (3.2) is unique of $\Lambda$ has nonempty interior, then the family of $\sigma$-periodic solutions to (3.2) is nonempty and compact in $\mathscr{B} \mathcal{C}([0, \infty) ; X)$.

Proof. The assertions follow if

$$
\lim _{h \rightarrow 0+}\left|x+h[B(t, x)+C(t, x)] ; K(\Lambda)_{R}\right| / h=0
$$

for all $(t, x) \in[0, \infty) \times K(\Lambda)_{R}$. Let $(t, x) \in[0, \infty) \times K(\Lambda)_{R}$. If $|x|<R$ then (3.3) follows from Lemmas 5 and 6. Suppose $|x|=R$ and let $\varepsilon>0$. For sufficiently small $h>0$ by Lemma 5 there exists $x_{h} \in K(\Lambda)$ for which $\left|x+h B(t, x)-x_{h}\right| \leqslant h \varepsilon / 2$. Let $y_{h}=x_{h}+h C(t, x) \in K(\Lambda)$. If $\left|y_{h}\right|<R$ then $y_{h} \in K(\Lambda)_{R}$ and we are done. Suppose $\left|y_{h}\right|>R$. By the Hahn-Banach Theorem there exist for each $h>0, \phi_{h} \in X^{*}$ such that

$$
\left|\phi_{h}\right|=1
$$

and

$$
\phi_{h}(x+h[B(t, x)+C(t, x)])=|x+h[B(t, x)+C(t, x)]| .
$$

Thus, there exist $h_{n}>0$ with $\lim _{h \rightarrow \infty} h_{n}=0$ and $\phi \in X^{*}$ such that $\lim _{n \rightarrow \infty} \phi_{h_{n}}$ $=\phi$ in the weak star topology. Since $\langle B(t, x)+C(t, x), x\rangle_{+} \leqslant 0$ we have 


$$
\begin{aligned}
\phi(B(t, x)+C(t, x))<0 \text { and } & \\
\left|x+h_{n}(B(t, x)+C(t, x))\right| & =\phi_{h_{n}}\left(x+h_{n}(B(t, x)+C(t, x))\right) \\
& =\phi_{h_{n}}(x)+h_{n} \phi_{h_{n}}(B(t, x)+C(t, x)) \\
& \leqslant \phi_{h_{n}}(x)+h_{n} \varepsilon / 2 \leqslant|x|+h_{n} \varepsilon=R+h_{n} \varepsilon
\end{aligned}
$$

for $\boldsymbol{n}$ sufficiently large. Consequently,

$$
\begin{aligned}
\left|y_{h_{n}}\right| & \leqslant\left|x+h_{n}(B(t, x)+C(t, x))\right|+\left|y_{h_{n}}-x-h_{n}(B(t, x)+C(t, x))\right| \\
& \leqslant R+h_{n} \varepsilon / 2
\end{aligned}
$$

and

$$
\begin{aligned}
\mid x+ & h_{n}(B(t, x)+C(t, x)) ; K(\Lambda)_{R} \mid \\
& <\left|x+h_{n}(B(t, x)+C(t, x))-y_{h_{n}}\right|+\left|y_{h_{n}}-\left(R /\left|y_{h_{n}}\right|\right) y_{h_{n}}\right| \\
& <h_{n} \varepsilon .
\end{aligned}
$$

Also, since $K(\Lambda)_{R}$ is convex the map $h \rightarrow \mid x+h(B(t, x)+C(t, x))$; $K(\Lambda)_{k} \mid / h$ is decreasing as $h \rightarrow 0+$ and (3.3) follows for $|x|=R$.

Our final theorem illustrates application of the results.

Proposition 5. Let $z \in \mathcal{C}([0,1] ; \mathbf{R})$ such that $z(s) \geqslant 0$ for all $s \in[0,1]$. Suppose $g:[0, \infty) \times[0,1] \times[0,1] \times[0, \infty) \rightarrow \mathbf{R}$ is continuous with $g(t, s, r, \xi)$ $>0$ for all $(t, s, r, \xi)$ and $\lim _{\xi \rightarrow \infty}[g(t, s, r, \xi)] / \xi^{3}=0$ uniformly on compact subsets of $[0, \infty) \times[0,1] \times[0,1]$. Then there exist $R>0$ and a continuous function $u:[0, \infty) \times[0,1] \rightarrow \mathbf{R}$ such that $0 \leqslant u(t, s) \leqslant R$ and

$$
\frac{\partial u}{\partial t}(t, s)=-[u(t, s)]^{3}+\int_{0}^{1} g(t, s, r, u(t, r)) d r
$$

for all $(t, s) \in[0, \infty) \times[0,1]$ with $u(0, \cdot)=z(\cdot)$. If, in addition, $g$ is o-periodic in then there exists a $\sigma$-periodic solution to (3.4).

RemarK 3. Under the suppositions of Proposition 5, Theorem 5 applies to the equation $[z(s)]^{3}=\int_{0}^{1} g(s, r, z(r)) d r, 0 \leqslant z(s) \leqslant R$.

Acknowledgements. This paper is taken from the author's dissertation. The author is most appreciative to his advisor, R. H. Martin, Jr., for his guidance during the preparation of his dissertation.

\section{REFERENCES}

1. A. Cellina, On the local existence of solutions of ordinary differential equations, Polon. Sci. Bull. Acad. 4 (1972), 293-396.

2. M. G. Crandall and A. Pazy,Nonlinear evolution equations in Banach spaces, Israel J. Math. 11 (1972), 57-94.

3. T. Kato, Nonlinear semigroups and evolution equations, J. Math. Soc. Japan 19 (1967), 508-520. 
4. M. A. Krasnoselskii, Topological methods in the theory of nonlinear integral equations, Macmillan, New York, 1974.

5. __ Translation along trajectories of differential equations, Transl. Math. Monographs, Vol. 29, Amer. Math. Soc., Providence, R. I., 1968.

6. K. Kuratowski, Topology, Vol. 1, Academic Press, New York, 1966.

7. V. Lakshmikantham and S. Leela, Differential and integral inequalities, Vol. I, Academic Press, New York, 1969.

8. A. Lasota and J. A. Yorke, The generic property of existence of solutions of differential equations in Banach spaces, J. Differential Equations 13 (1973), 1-12.

9. T. Y. Li, Existence of solutions for ordinary differential equations, J. Differential Equations 18 (1975), 29-40.

10. R. H. Martin, Differential equations on closed subsets of a Banach space, Trans. Amer. Math. Soc. 179 (1973), 339-414.

11. Approximation and existence of solutions to ordinary differential equations in Banach spaces, Funkcial. Ekvac. 16 (1973), 195-211.

12. L Remarks on ordinary differential equations involving dissipative and compact operators, J. London Math. Soc. 10 (1975), 61-65.

13. Invariant sets for perturbed semigroups of linear operators, Ann. Mat. Pura Appl. 105 (1975), 221-239.

14. B. N. Sadovskii, On a fixed point principle, Functional Anal. Appl. 1 (1967), 74-76.

Department of Mathematics, North Carolina State University, Raleigh, North CAROLINA 27607

Current address: Department of Mathematics, Pan American Univeristy, Edinburg, Texas 78539 